\title{
720 CUE-102 SELECTIVELY ACTIVATES AND EXPANDS WT1- SPECIFIC T CELLS FOR THE TREATMENT OF PATIENTS WITH WT1+ MALIGNANCIES
}

${ }^{1}$ Christie Zhang*, 'Natasha Girgis, 'Zohra Merazga, 'Steven Hatfield, 'Alex Histed, ${ }^{1}$ Fan Zhao, ${ }^{1}$ Raymond Moniz, ${ }^{1}$ Kristin Yeung, ${ }^{1}$ Fulvio Diaz, ${ }^{1}$ Jason Brown, ${ }^{1}$ Mark Haydock, ${ }^{1}$ Luke Witt, ${ }^{1}$ Wynona Bautista, ${ }^{1} J o h n$ Ross, ${ }^{1}$ Saso Cemerski, 'Anish Suri, ${ }^{2}$ Kenneth Pienta, ${ }^{1}$ Matteo Levisetti, 'Steve Quayle. 'Cue Biopharma, Cambridge, MA, United States; ${ }^{2} J o h n s$ Hopkins School of Medicine, Cambridge, MA, United States

Background Wilms' Tumor 1 (WT1) was ranked as the highest priority antigen for therapeutic targeting in an effort by the National Cancer Institute. Development of novel modalities targeting WT1 provide a significant opportunity to address high unmet medical need in WT1-positive malignancies, including AML, ovarian, endometrial, breast, lung, colorectal and pancreatic cancer. Leveraging the Immuno-STAT platform of targeted IL-2 therapies, and the ongoing development of CUE-101, CUE-102 is being developed as a novel therapeutic fusion protein to selectively activate tumor antigen-specific $\mathrm{T}$ cells to treat WT1-expressing cancers. CUE-102 consists of two human leukocyte antigen (HLA) molecules presenting a WT1 peptide, four affinity-attenuated human interleukin-2 (IL2) molecules, and an effector attenuated human immunoglobulin $\mathrm{G}$ (IgG1) Fc domain.

Methods Human PBMCs were tested to demonstrate cellular activity and specificity of CUE-102, while in vivo activity of CUE-102 was assessed in HLA-A2 transgenic mice. HLA-A2/ WT1-specific TCRs were validated and expressed in primary human CD8 $\mathrm{T}$ cells. Tetramer staining and flow cytometry identified cell populations and activation markers.

Results Multiple in vitro assessments demonstrate that CUE102 selectively activates and expands WT1-specific CD8 + T cells from PBMC of healthy and cancer bearing donors. These CUE-102-expanded CD8 $+\mathrm{T}$ cells exhibit polyfunctional and cytotoxic responses upon challenge with WT1-presenting target cells. In addition, significant functional attenuation of the IL-2 components of CUE-102 was shown, similar to preclinical results obtained with CUE-101. In vivo studies in HLA-A2 transgenic mice confirm that CUE-102 elicits and expands polyfunctional WT1-specific CD8 $+\mathrm{T}$ cells from naïve and previously immunized mice without significantly altering the frequencies of other immune lineages. The WT1-specific CD8 $+\mathrm{T}$ cells expanded in vivo exhibit polyfunctionality in response to peptide-loaded target cells, and selectively kill WT1-presenting target cells in vivo.

Conclusions CUE-102 elicits selective expansion of a WT1-specific population of cytotoxic CD8 $+\mathrm{T}$ cells both in vitro and in vivo. These results, together with its similarity to CUE-101, support its anticipated tolerability profile and potential for clinical efficacy in a Phase 1 trial planned to initiate in 2022.

Ethics Approval All animal studies followed guidance from the SmartLabs Institutional Animal Care and Use Committee protocol MIL-100 and were performed in compliance with federal guidelines.

http://dx.doi.org/10.1136/jitc-2021-SITC2021.720 